\title{
A institucionalização da seleção de medicamentos em hospitais públicos por meio do planejamento estratégico situacional
}

\author{
Rafael Santos Santana \\ Universidade de Brasília \\ Iza Maria Fraga Lobo \\ Universidade Federal de Sergipe \\ Thais Rodrigues Penaforte \\ Universidade Federal da Bahia \\ Silvana Nair Leite \\ Universidade Federal de Santa Catarina \\ Wellington Barros Silva \\ Universidade Federal de Sergipe
}

\begin{abstract}
Desde a publicação da primeira relação de medicamentos essenciais da Organização Mundial da Saúde (OMS), em 1977, essa instituição de saúde e diversas outras em todo o mundo têm fomentado a importância da promoção de políticas de medicamentos essenciais para os serviços de saúde. Apesar da inegável contribuição para a promoção do uso racional de medicamentos, a implantação de comitês que gerenciem a implantação de listas de medicamentos essenciais ainda é um desafio para os gestores do Sistema Único de Saúde (SUS). Este trabalho teve como objetivo utilizar uma ferramenta de gestão, proposta pelo economista Carlos Matus, para a superação das dificuldades de implantação de Comissões de Farmácia e Terapêutica nos serviços hospitalares do estado de Sergipe. Para isso, a equipe de pesquisadores realizou, em conjunto com os atores do serviço, a implantação do Planejamento Estratégico Situacional (PES) por meio dos momentos explicativo, normativo, estratégico e tático-operacional. Por meio deste trabalho, observou-se que o método PES caracteriza-se como uma ferramenta recomendada para a implantação de atividades fundamentais da seleção de medicamentos, agrupadas em três objetivos gerais alcançados: 1 ) a regulamentação de fluxos e procedimentos para a seleção de medicamentos; 2) a organização de comissões de farmácia e terapêutica e 3) a elaboração de listas de medicamentos essenciais.
\end{abstract}

Palavras-chave: seleção de medicamentos; avaliação de serviços de saúde; uso racional de medicamentos; planejamento estratégico situacional.

DOI: http://dx.doi.org/10.1590/0034-76121596

Artigo recebido em 8 jun. 2013 e aceito em 26 jun. 2014.

Rev. Adm. Pública - Rio de Janeiro 48(6):1587-1603, nov./dez. 2014 
La institucionalización del proceso de selección de medicamentos en los hospitales públicos a través de planificación estratégica situacional

Desde la publicación de la primera lista modelo de la Organización Mundial de la Salud (OMS) de medicamentos esenciales en 1977, este órgano y varias instituciones de salud en todo el mundo han promovido la importancia de la promoción de políticas de medicamentos esenciales a los servicios de salud. A pesar de la innegable contribución a la promoción del uso racional de los medicamentos, la implementación de los comités que administran la aplicación de listas de medicamentos esenciales sigue siendo un reto para los gestores en el Sistema Único de Salud de Brasil. Este estudio tuvo como objetivo utilizar una herramienta de gestión, propuesta por el economista Carlos Matus, para superar las dificultades de implantación del comité de farmacia y terapéutica en servicios hospitalarios en el estado de Sergipe. Para ello, el equipo de investigación ante los actores del servicio aplicaron la Planificación Estratégica Situacional (PES) por medio de los momentos explicativo, normativo, estratégico y táctico-operacional. A través de este trabajo, se observó que el método PES se caracteriza por ser una herramienta recomendable para la ejecución de las actividades clave de la selección de medicamentos, en este estudio, agrupadas en tres objetivos generales alcanzados: 1) la regulación de los flujos y procedimientos para selección de medicamentos, 2) la organización de comités de farmacia y terapéutica, y 3) la preparación de las listas de medicamentos esenciales.

Palabras clave: selección de medicamentos; evaluación de servicios de salud; uso racional de medicamentos; planificación estratégico situacional.

Institutionalization of medicine selection in public hospitals through the situational strategic planning

Since the publication of the first list of World Health Organization (WHO)'s essential medicines, in 1977, this one and some health institutions around the world have encouraged the importance of promoting policies of essential medicines for health services. Despite the undeniable contribution to the promotion of rational use of medicines, the implementation of committees that manage the deployment of lists of essential medicines is still a challenge for managers of the Brazilian health system (SUS). This study aimed to use a management tool proposed by economist Carlos Matus to overcome the difficulties of implementing pharmacy and therapeutic committees in hospital services of Sergipe, State of Brazil. To do it, the research team conducted with the stakeholders of the implementation of the Situational Strategic Planning (PES) through explanatory, normative, strategic and tactical-operational moments. It was observed that the PES method is characterized as a recommended tool for the implementation of fundamental activities of of essential medicines' selection, in this study, grouped into three general objectives achieved: 1) the regulation of flows and procedures for selecting medication, 2) the organization of pharmacy and therapeutic committees, and 3) the preparation of lists of essential medicines.

KEYWoRDs: selection of medicines; assessment of health services; rational drug use; situational strategic planning.

\section{Introdução}

O elevado número de tecnologias produzidas e incorporadas nas últimas duas décadas tem sido associado à queda na mortalidade, ao aumento do volume de informação produzido e do custo da assistência em saúde (Krauss-Silva, 2003). Também é crescente a preocupação 
com a qualidade dos serviços prestados e com os recursos necessários para sua manutenção e expansão. O medicamento, uma tecnologia muito importante nesse processo, foco de intensa inovação, reflete muito bem a necessidade de constantes avaliações para que o seu uso seja benéfico à saúde, mas principalmente custo-efetivo e resolutivo (Marques e Zucchi, 2006).

Essas são razões para incorporar os critérios de promoção do uso racional de medicamentos nos modelos de gestão e prestação de serviços em saúde, aí incluída a avaliação para a incorporação e substituição de tecnologias para a saúde. A premência dos motivos de ordem ética, econômica e social justifica tal necessidade: o tratamento de um indivíduo deve oferecer o máximo benefício com o mínimo de risco e, ao mesmo tempo, a sociedade deve maximizar os recursos financeiros (Velásquez, 1999).

Os gastos crescentes nos últimos anos no setor saúde vêm preocupando diversos países, em especial aqueles cuja atenção à saúde é garantida pelo setor público. A grande pressão pela incorporação de tecnologias - incluindo procedimentos, produtos para a saúde e medicamentos - cada vez mais caras, além de impactar no orçamento dos países, produz uma restrição do acesso aos serviços de saúde, visto que não é possível garantir, a todo mundo, todas as intervenções (Santos, 2010).

De uma forma geral, o gasto com medicamentos representa cerca de 30 a $40 \%$ do orçamento em saúde (Marques e Zucchi, 2006). No Brasil, o Sistema Único de Saúde (SUS) é um grande incorporador de tecnologias. Somente o Ministério da Saúde compra cerca de R 8 bilhões em medicamentos e produtos para saúde por ano. Ao mesmo tempo, gestores de todas as instâncias do SUS são constantemente pressionados para que tecnologias novas e emergentes sejam incorporadas. Grande parte dessa pressão é norteada pelo desconhecimento de sua viabilidade técnica, financeira e das consequências do seu uso para a saúde da população (Silva, Petramale e Elias, 2012).

A principal alternativa a essa problemática é a definição das listas de medicamentos essenciais. Já em 1975, a Assembleia Mundial da Saúde sugeriu que a OMS deveria assessorar seus estados-membros a selecionar medicamentos essenciais com qualidade e custo razoável. Desse modo, em 1977, publicou-se a primeira lista modelo de medicamentos essenciais com 205 itens (186 medicamentos). Desde então, 17 revisões foram publicadas, e a maioria dos países-membros adota listas de medicamentos essenciais (Wannmacher, 2010; WHO, 2013).

O conceito de medicamentos essenciais tem como objetivo primordial fornecer condições para contemplar as necessidades da terapêutica e melhorar a qualidade de assistência, e não o de restringir a oferta de terapias. Selecionam-se medicamentos levando em conta sua pertinência para a saúde pública, provas de sua eficácia e segurança, e efetividade comparativa em relação ao custo. Os medicamentos essenciais devem estar disponíveis nos sistemas de saúde em todo momento, em quantidades suficientes, nas formas farmacêuticas apropriadas, com garantia da qualidade e informação adequada, a um preço que os pacientes e a comunidade possam pagar (Wannmacher, 2010; WHO, 2002).

No âmbito hospitalar e dos sistemas locais de saúde, o processo de elaboração da lista de medicamentos essenciais é realizado por uma equipe multidisciplinar denominada, geralmente, de Comissão de Farmácia e Terapêutica (CFT), cujo trabalho está pautado sob a 
ótica da Saúde Baseada em Evidências. No Brasil, embora haja a recomendação, não existe a obrigatoriedade da existência desses comitês nos serviços de saúde, como acontece com as Comissões de Controle de Infecção Hospitalar (CCIH) (Marques e Zucchi, 2006), o que talvez explique sua pouca implantação nos hospitais brasileiros. De 250 hospitais avaliados no país por Osório e Castilho (2004), 139 hospitais possuíam CCIH e apenas 29 hospitais possuíam CFT (Osório e Castilho, 2004).

A maioria das CFT localiza-se em países desenvolvidos, incluindo Austrália, EUA e países europeus. Na Austrália e no Reino Unido, 92\% e 86\% dos hospitais, respectivamente, desenvolviam, em 1990, algum tipo de comissão terapêutica hospitalar. Nos EUA, o recebimento de acreditação hospitalar é condicionado à existência de CFT ou comissão similar (Weekes e Brooks, 1996; Cebrim, 2003). As experiências exitosas no Brasil precisam ser divulgadas e avaliadas, poucos são os trabalhos que demonstram as formas de atuação e os resultados obtidos por CFT em instituições nacionais.

As dificuldades de implantação de CFT e a manutenção de suas funções se tornam um desafio para os gestores dos serviços de saúde. Esse desafio é ainda maior quando se verifica o forte caráter normativo nos planos e gestão da saúde. O usual planejamento normativo (PN) é uma proposta tecnocrática, onde o planejador, baseado em seus conhecimentos técnicos "neutros", faz o plano e estabelece prioridades. É um método normativo e economicista, onde a norma - o deve ser - tem como fundamento principal a relação custo-benefício (Giovanella, 1991).

Uma alternativa a esse modelo é a proposta do planejamento estratégico situacional (PES), projetado para a administração pública, com grande potencial para processar problemas complexos. Esse tipo de planejamento, ao contrário do PN, se caracteriza por levar em consideração múltiplas dimensões para avaliação dos problemas (política, econômica, social) e análise estratégica dos diferentes atores envolvidos em uma situação de partilha de poder, quadro comum em serviços geridos pelo governo (Matus, 1996). Suas características principais envolvem o processo participativo e interativo entre todos os atores envolvidos no serviço, a negociação e pactuação de ações e responsabilidades com base na análise da situação real.

Constatada a necessidade de mudança no processo de gestão da assistência farmacêutica na rede hospitalar de Sergipe, foi aplicado o método PES para promover a implantação de CFT e estabelecer uma política de seleção de medicamentos baseada em critérios científicos. O presente estudo visa demonstrar a experiência e seus resultados nos hospitais sergipanos.

\section{Métodos}

O trabalho é resultante do exercício de construção de um plano operativo de intervenção no serviço proposto pelo Curso de Especialização em Gestão da Assistência Farmacêutica do Ministério da Saúde (Leite e Guimarães, 2011). Trata-se de um estudo de caso com abordagem metodológica da pesquisa avaliativa em saúde, de caráter observacional, com enfoque quantitativo e qualitativo complementares. Esse tipo de metodologia é característico das pes- 
quisas voltadas para a avaliação de serviços e processos de saúde por conta da complexidade do fenômeno social investigado (Contradiopoulos, 1997; Minayo, 1993). O fato de parte dos pesquisadores ser também gestora dos serviços foi decisivo para a elaboração deste trabalho e construção dos respectivos planos operativos.

O campo da pesquisa foi uma rede composta de 12 hospitais públicos, com porte e especialidades diferentes, e pelo Serviço de Atendimento Móvel de Urgência estadual, ambos geridos por uma fundação estadual pública de direito privado. Todas as unidades incluídas, até o início do estudo, não possuíam nenhum processo padronizado de seleção de medicamentos e incorporação de tecnologias. Também não foi constatada a existência de lista de medicamentos publicada oficialmente.

De janeiro de 2011 a junho de 2012 foi acompanhado o processo de implantação das atividades de seleção de medicamentos por meio do método PES, proposto pelo economista chileno Carlos Matus (1996).

\section{Resultados e discussão}

Conforme o modelo de PES, os trabalhos foram baseados nos momentos: explicativo, normativo, estratégico e tático-operacional, que fazem parte da construção de um plano operativo, neste caso com o objetivo final de instituir uma política de seleção de medicamentos nas unidades de saúde-alvo do estudo.

O conceito de momento, formulado para superar a ideia de "etapas rígidas", implica uma visão dinâmica do processo de planejamento, que se caracteriza pela permanente interação de suas fases e pela constante retomada destas (Artmann, 1997).

Figura 1

Momentos propostos pelo modelo PES

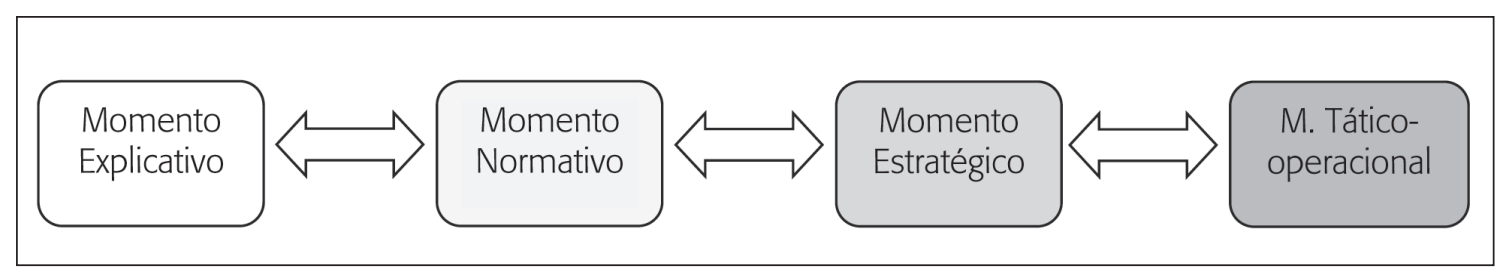

Fonte: Adaptada de Matus (1996).

Durante todo o processo foram realizados registros das ações e, para avaliação dos resultados, recorreu-se à análise documental de atas de reuniões, memorandos, pareceres técnicos e demais documentos produzidos.

Na fase inicial da pesquisa foi realizada uma avaliação dos locais de estudo, que possibilitou identificar o perfil dos serviços, descritos no quadro 1. 
Quadro 1

Características dos serviços de saúde avaliados

\begin{tabular}{|ccc|}
\hline CARACTERÍSTICAS & TIPO & Frequência \\
\hline \multirow{2}{*}{ Natureza jurídica } & Público & $100 \%(12)$ \\
& Privado & $0,0 \%(0)$ \\
\hline Serviços e Grau de & Hospitais locais (até 40 leitos) & $30,7 \%(04)$ \\
Complexidade & Hospitais regionais (40 a 150 leitos) & $38,4 \%(05)$ \\
& Serviço exclusivo de maternidade & $15,3 \%(02)$ \\
& Hospital de grande porte com especialidades (600 leitos) & $7,6 \%(01)$ \\
& Serviço de atendimento pré-hospitalar & $7,6 \%(01)$ \\
\hline
\end{tabular}

Fonte: Resultados da pesquisa.

\subsection{Momento explicativo}

Este é o momento em que se analisa a realidade presente e o hiato existente com o futuro desejado. Foram identificados e selecionados os problemas, explicando com profundidade as causas de cada um e do seu conjunto.

A partir de oficinas de avaliação da assistência farmacêutica, que contaram com participação de "atores-chave" do processo (farmacêuticos, médicos, enfermeiros, gestores), foi possível discutir os principais problemas encontrados, categorizá-los e avaliá-los quanto à sua magnitude, transcendência, factibilidade e vulnerabilidade, culminando com a definição do problema prioritário, definido pelos componentes da oficina como: "Não regularização do processo de seleção de medicamentos".

O problema priorizado foi trabalhado pela equipe que definiu os descritores, causas e consequências deste, seguindo o modelo de diagrama de causa e efeito de Ishikawa (1993), conhecido como diagrama "espinha de peixe".

\subsection{Momento normativo}

Neste momento é delineado o plano de intervenção. O momento normativo caracteriza-se pela definição da imagem objetivo ou situação futura desejada e das operações/ações concretas que visam solucionar os nós críticos do processo (Bernardino, 2006). Foi possível realizar um diagnóstico mais detalhado do quadro situacional e das modificações necessárias para alcançar a imagem-objetivo definida pelos profissionais do serviço: "Institucionalização do processo de seleção de medicamentos nos hospitais da rede". No quadro 2 pode-se verificar detalhadamente os dados deste momento. 


\subsection{Momento estratégico}

Neste momento é realizada a análise da possibilidade de execução do plano em suas várias dimensões: política, econômica, competência técnica e administrativa. A análise situacional encontra-se focada na construção da viabilidade, mapeando-se todos os atores que possam cooperar ou se opor ao que está sendo proposto, avaliando-se o tipo de controle que cada um detém dos recursos essenciais para o êxito do plano (Almeida et al., 2001; Melleiro et al., 2005). No quadro 3 está descrita a análise de viabilidade e factibilidade realizada para alcance dos objetivos propostos, na perspectiva da gestão da assistência farmacêutica que liderou o processo de implantação do PES. No final desse momento foi possível observar as atividades estratégicas principais que deveriam ser executadas pela gestão e demais atores do serviço para sucesso do planejamento e implantação efetiva da seleção de medicamentos.

Quadro 2

Matriz do momento normativo: definição de objetivos, operações e ações

\begin{tabular}{|c|c|c|}
\hline OBJETIVOS & OPERAÇÕES & AÇÕES ESPECÍFICAS \\
\hline \multirow{6}{*}{$\begin{array}{l}\text { 1. Regulamentar fluxos } \\
\text { e procedimentos } \\
\text { de incorporação de } \\
\text { tecnologias em saúde } \\
\text { (medicamentos) }\end{array}$} & \multirow{3}{*}{$\begin{array}{l}\text { 1.1 Formação de grupo de trabalho } \\
\text { para definição dos padrões de } \\
\text { seleção de tecnologias }\end{array}$} & Contato com atores-chave para grupo \\
\hline & & Elaboração de minuta da resolução \\
\hline & & Discussão de fluxos e rotinas no grupo \\
\hline & \multirow{3}{*}{$\begin{array}{l}\text { 1.2 Elaboração de resolução com } \\
\text { normas para incorporação de } \\
\text { medicamentos }\end{array}$} & Solicitação de parecer jurídico \\
\hline & & Redação de documento final \\
\hline & & Validação pela direção da instituição \\
\hline \multirow{5}{*}{$\begin{array}{l}\text { 2. Organizar CFT de } \\
\text { acordo com o nível de } \\
\text { complexidade dos serviços }\end{array}$} & \multirow{2}{*}{$\begin{array}{l}\text { 2.1 Regulamentação das CFT na } \\
\text { instituição }\end{array}$} & $\begin{array}{l}\text { Divulgação da resolução com enfoque nos fluxos e rotinas } \\
\text { da CFT }\end{array}$ \\
\hline & & Indicação de membros representativos para compor as CFT \\
\hline & \multirow{3}{*}{$\begin{array}{c}\text { 2.2 Formação das comissões, } \\
\text { nomeação de membros e início } \\
\text { dos trabalhos }\end{array}$} & $\begin{array}{l}\text { Posse dos membros e assinatura de declaração de } \\
\text { conflito de interesses }\end{array}$ \\
\hline & & $\begin{array}{l}\text { Definição de atribuições entre os membros e elaboração } \\
\text { de cronograma de reuniões }\end{array}$ \\
\hline & & $\begin{array}{l}\text { Treinamento dos membros das CFT sobre avaliação de } \\
\text { tecnologias em saúde }\end{array}$ \\
\hline \multirow{6}{*}{$\begin{array}{l}\text { 3. Elaborar relações de } \\
\text { medicamentos essenciais } \\
\text { de acordo com nível de } \\
\text { complexidade do serviço }\end{array}$} & \multirow{3}{*}{$\begin{array}{l}\text { 3.1 Seleção dos medicamentos } \\
\text { essenciais pelas CFT }\end{array}$} & $\begin{array}{l}\text { Formatação de lista inicial baseada nos registros de } \\
\text { compra e estoque das unidades }\end{array}$ \\
\hline & & Revisão dos itens por grupo terapêutico \\
\hline & & Elaboração das listas com itens selecionados pelas CFT \\
\hline & \multirow{3}{*}{$\begin{array}{l}\text { 3.2. Validação das listas de } \\
\text { medicamentos selecionados }\end{array}$} & Consulta pública da lista selecionada \\
\hline & & Apreciação das contribuições e julgamentos finais das CFT \\
\hline & & $\begin{array}{l}\text { Institucionalização das listas de medicamentos e das } \\
\text { normas para alteração dos itens posteriormente }\end{array}$ \\
\hline
\end{tabular}

Fonte: Resultados da pesquisa. 


\subsection{Momento tático-operacional}

Este é o momento de colocar o PES em prática. Nessa fase, as avaliações mais importantes estavam relacionadas ao impacto da implantação do plano no serviço e a atual conjuntura da organização.

Temas vitais abordados nesse momento foram a estrutura organizacional, o fluxo interno de informações, a coordenação do plano, do sistema de prestação de contas, as ferramentas gerenciais necessárias e, finalmente, a dinâmica da participação democrática na implantação do plano. É importante destacar que o planejamento estratégico só termina quando é executado, é o oposto à visão tradicional do "plano-livro" que, separando planejadores dos executores, estabelece uma dicotomia insuperável entre o conhecer e o agir (Toni, 2004).

O quadro 4 descreve a matriz do momento tático-operacional construída para implantação do processo de seleção e define os atores envolvidos na execução das atividades específicas, prazos de execução e respectivos indicadores de avaliação.

$$
\text { Quadro } 3
$$

Matriz do momento estratégico: análise da viabilidade e factibilidade.

\begin{tabular}{|c|c|c|c|c|c|c|c|c|}
\hline \multirow{2}{*}{ OBJETIVOS } & \multirow{2}{*}{ OPERAÇÕES } & \multirow{2}{*}{ AÇÕES ESPECÍFICAS } & \multicolumn{3}{|c|}{$\begin{array}{l}\text { ANÁLISE DE VIABILIADADE } \\
\text { (Poder) }\end{array}$} & \multicolumn{2}{|c|}{$\begin{array}{c}\text { ANÁLISE DE } \\
\text { FACTIBILIDADE }\end{array}$} & \multirow{2}{*}{$\begin{array}{l}\text { ATIVIDADE } \\
\text { ESTRATÉGICA }\end{array}$} \\
\hline & & & Decidir & Executar & Manter & $\begin{array}{l}\text { Recursos } \\
\text { Existentes }\end{array}$ & $\begin{array}{l}\text { Recursos } \\
\text { Necessários }\end{array}$ & \\
\hline \multirow{6}{*}{$\begin{array}{l}\text { 1. Regulamentar } \\
\text { fluxos e } \\
\text { procedimentos } \\
\text { de incorporação } \\
\text { de tecnologias } \\
\text { em saúde } \\
\text { (medicamentos) }\end{array}$} & \multirow{3}{*}{$\begin{array}{l}1.1 \text { Formação de } \\
\text { grupo de trabalho } \\
\text { para definição dos } \\
\text { padrões de seleção } \\
\text { de tecnologias }\end{array}$} & $\begin{array}{l}\text { Contato com atores-chave } \\
\text { para GT }\end{array}$ & $\operatorname{sim}$ & $\operatorname{sim}$ & $\operatorname{sim}$ & Equipe & - & \multirow{6}{*}{$\begin{array}{c}\text { Articular } \\
\text { parceria com } \\
\text { - membros da } \\
\text { gestão e das } \\
\text { unidades } \\
\text { para formar } \\
\text { grupo de } \\
\text { trabalho } \\
\text { inicial }\end{array}$} \\
\hline & & $\begin{array}{l}\text { Elaboração de minuta da } \\
\text { resolução }\end{array}$ & $\operatorname{sim}$ & $\operatorname{sim}$ & $\operatorname{sim}$ & $\begin{array}{c}\text { Equipe } \\
\text { e TI }\end{array}$ & - & \\
\hline & & Discussão de fluxos e rotinas & $\operatorname{sim}$ & $\operatorname{sim}$ & $\operatorname{sim}$ & Equipe & & \\
\hline & \multirow{3}{*}{$\begin{array}{l}1.2 \text { Elaboração } \\
\text { de resolução } \\
\text { com normas para } \\
\text { incorporação de } \\
\text { medicamentos }\end{array}$} & $\begin{array}{l}\text { Solicitação de parecer } \\
\text { jurídico }\end{array}$ & $\operatorname{sim}$ & não & não & -- & $\begin{array}{l}\text { Atores } \\
\text { externos }\end{array}$ & \\
\hline & & Redação de documento final & $\operatorname{sim}$ & $\operatorname{sim}$ & $\operatorname{sim}$ & Equipe & & \\
\hline & & $\begin{array}{l}\text { Validação pela direção da } \\
\text { instituição }\end{array}$ & não & não & não & -- & $\begin{array}{l}\text { Atores } \\
\text { externos }\end{array}$ & \\
\hline \multirow{5}{*}{$\begin{array}{l}\text { 2. Organizar CFT } \\
\text { nas unidades de } \\
\text { estudo de acordo } \\
\text { com o nível de } \\
\text { complexidade do } \\
\text { serviço }\end{array}$} & \multirow{2}{*}{$\begin{array}{l}2.1 \\
\text { Regulamentação } \\
\text { das CFT na } \\
\text { instituição }\end{array}$} & $\begin{array}{l}\text { Divulgação da resolução com } \\
\text { enfoque nos fluxos e rotinas } \\
\text { da CFT }\end{array}$ & $\operatorname{sim}$ & $\operatorname{sim}$ & $\operatorname{sim}$ & $\begin{array}{l}\text { Equipe } \\
\text { e TI }\end{array}$ & $\begin{array}{l}\text { Material } \\
\text { Divulgação }\end{array}$ & \multirow{5}{*}{$\begin{array}{c}\text { Organizar } \\
\text { CFT, rotina } \\
\text { de trabalho e } \\
\text { treinamento } \\
\text { dos } \\
\text { membros }\end{array}$} \\
\hline & & $\begin{array}{l}\text { Indicação de membros para } \\
\text { CFT }\end{array}$ & $\operatorname{sim}$ & não & não & - & $\begin{array}{l}\text { Atores } \\
\text { externos }\end{array}$ & \\
\hline & \multirow{3}{*}{$\begin{array}{l}2.2 \text { Formação } \\
\text { das comissões, } \\
\text { nomeação de } \\
\text { membros e início } \\
\text { dos trabalhos }\end{array}$} & $\begin{array}{l}\text { Posse dos membros e } \\
\text { assinatura de declaração de } \\
\text { conflito de interesses }\end{array}$ & $\operatorname{sim}$ & $\operatorname{sim}$ & $\operatorname{sim}$ & -- & -- & \\
\hline & & $\begin{array}{l}\text { Definição de atribuições } \\
\text { entre os membros e } \\
\text { cronograma de reuniões }\end{array}$ & $\operatorname{sim}$ & $\operatorname{sim}$ & $\operatorname{sim}$ & - & -- & \\
\hline & & $\begin{array}{l}\text { Treinamento dos membros } \\
\text { sobre avaliação de } \\
\text { tecnologias em saúde }\end{array}$ & $\operatorname{sim}$ & não & não & Equipe & $\begin{array}{l}\text { Recursos } \\
\text { financeiros }\end{array}$ & \\
\hline
\end{tabular}




\begin{tabular}{|c|c|c|c|c|c|c|c|c|}
\hline \multirow{2}{*}{ OBJETIVOS } & \multirow{2}{*}{ OPERAÇÕES } & \multirow{2}{*}{ AÇÕES ESPECÍFICAS } & \multicolumn{3}{|c|}{$\begin{array}{l}\text { ANÁLISE DE VIABILIADADE } \\
\text { (Poder) }\end{array}$} & \multicolumn{2}{|c|}{$\begin{array}{l}\text { ANÁLISE DE } \\
\text { FACTIBILIDADE }\end{array}$} & \multirow{2}{*}{$\begin{array}{c}\text { ATIVIDADE } \\
\text { ESTRATÉGICA }\end{array}$} \\
\hline & & & Decidir & Executar & Manter & $\begin{array}{l}\text { Recursos } \\
\text { Existentes }\end{array}$ & $\begin{array}{l}\text { Recursos } \\
\text { Necessários }\end{array}$ & \\
\hline \multirow{6}{*}{$\begin{array}{l}\text { 3. Elaborar } \\
\text { relações de } \\
\text { medicamentos } \\
\text { essenciais de } \\
\text { acordo com nível } \\
\text { de complexidade } \\
\text { do serviço }\end{array}$} & \multirow{3}{*}{$\begin{array}{c}\text { 3.1 Seleção dos } \\
\text { medicamentos } \\
\text { essenciais pelas } \\
\text { CFT }\end{array}$} & $\begin{array}{l}\text { Elaboração de lista inicial } \\
\text { baseada no histórico de } \\
\text { utilização das unidades }\end{array}$ & $\operatorname{sim}$ & $\operatorname{sim}$ & $\operatorname{sim}$ & Equipe & - & \multirow{6}{*}{$\begin{array}{c}\text { Validar } \\
\text { Trabalho } \\
\text { da CFT e } \\
\text { divulgar as } \\
\text { ações }\end{array}$} \\
\hline & & $\begin{array}{l}\text { Revisão dos medicamentos } \\
\text { de acordo com grupo } \\
\text { terapêutico }\end{array}$ & $\operatorname{sim}$ & $\operatorname{sim}$ & $\operatorname{sim}$ & $\begin{array}{l}\text { Equipe, } \\
\text { Base de } \\
\text { dados }\end{array}$ & - & \\
\hline & & $\begin{array}{l}\text { Elaboração das listas com } \\
\text { itens selecionados pelas CFT }\end{array}$ & $\operatorname{sim}$ & $\operatorname{sim}$ & $\operatorname{sim}$ & $\begin{array}{c}\text { Equipe, } \\
\text { Base de } \\
\text { dados }\end{array}$ & -- & \\
\hline & \multirow{3}{*}{$\begin{array}{l}3.2 \text { Validação } \\
\text { das listas de } \\
\text { medicamentos } \\
\text { selecionados }\end{array}$} & $\begin{array}{l}\text { Consulta pública da lista } \\
\text { selecionada }\end{array}$ & $\operatorname{sim}$ & não & não & Equipe & $\begin{array}{l}\text { Material } \\
\text { Divulgação }\end{array}$ & \\
\hline & & $\begin{array}{l}\text { Apreciação das contribuiç̃̃es } \\
\text { e julgamentos finais das CFT }\end{array}$ & $\operatorname{sim}$ & $\operatorname{sim}$ & $\operatorname{sim}$ & Equipe & -- & \\
\hline & & $\begin{array}{l}\text { Institucionalização das Listas e } \\
\text { normas de alteração posterior }\end{array}$ & não & não & não & - & $\begin{array}{l}\text { Atores } \\
\text { externos }\end{array}$ & \\
\hline
\end{tabular}

Fonte: Resultados da Pesquisa

Quadro 4

Matriz do momento tático-operacional: atores, prazos e indicadores

\begin{tabular}{|c|c|c|c|c|c|c|}
\hline \multirow{2}{*}{ OBJETIVOS } & \multirow{2}{*}{ OPERAÇÕES } & \multirow{2}{*}{ AÇÕES ESPECÍFICAS } & \multicolumn{2}{|c|}{$\begin{array}{l}\text { RESPONSABILIDADE E } \\
\text { CENTRALIDADE }\end{array}$} & \multirow{2}{*}{ PRAZO } & \multirow{2}{*}{$\begin{array}{l}\text { INDICADOR } \\
\text { DE AVALIAÇÃO } \\
\text { [tempo } \\
\text { estimado] }\end{array}$} \\
\hline & & & $\begin{array}{c}\text { ATOR } \\
\text { PRINCIPAL }\end{array}$ & PARCEIROS & & \\
\hline \multirow{6}{*}{$\begin{array}{l}\text { 1. Regulamentar } \\
\text { fluxos e } \\
\text { procedimentos } \\
\text { de incorporação } \\
\text { de tecnologias } \\
\text { em saúde } \\
\text { (medicamentos) }\end{array}$} & \multirow{3}{*}{$\begin{array}{l}1.1 \text { Formação de } \\
\text { grupo de trabalho } \\
\text { (GT) para definição } \\
\text { dos padrões } \\
\text { de seleção de } \\
\text { tecnologias }\end{array}$} & $\begin{array}{l}\text { Contato com atores-chave para } \\
\text { grupo }\end{array}$ & Coord. AF & $\begin{array}{l}\text { Coord. Enf. e } \\
\text { Dir. Clínica }\end{array}$ & 7 dias & \multirow{6}{*}{$\begin{array}{c}\text { Publicação de } \\
\text { Resolução com } \\
\text { regulamentação } \\
\text { do processo de } \\
\text { seleção } \\
\text { [2 meses] }\end{array}$} \\
\hline & & Elaboração de minuta da resolução & $\begin{array}{l}\text { Farmacêutico } \\
\text { do GT }\end{array}$ & - & 15 dias & \\
\hline & & $\begin{array}{l}\text { Discussão de fluxos e rotinas no } \\
\text { grupo }\end{array}$ & $\begin{array}{l}\text { Membros } \\
\text { do GT }\end{array}$ & - & 7 dias & \\
\hline & \multirow{3}{*}{$\begin{array}{l}\text { 1.2 Elaboração } \\
\text { de resolução } \\
\text { com normas para } \\
\text { incorporação de } \\
\text { medicamentos }\end{array}$} & Solicitação de parecer jurídico & Procurador & - & 7 dias & \\
\hline & & Redação de documento final & Relator do GT & - & 15 dias & \\
\hline & & Validação pela direção da instituição & Coord. AF & $\begin{array}{l}\text { Coord. Enf. e } \\
\text { Dir. Clínica }\end{array}$ & 7 dias & \\
\hline \multirow{5}{*}{$\begin{array}{l}\text { 2. Organizar } \\
\text { comissões } \\
\text { de farmácia e } \\
\text { terapêutica nas } \\
\text { unidades de } \\
\text { estudo de acordo } \\
\text { com o nível de } \\
\text { complexidade do } \\
\text { serviço }\end{array}$} & \multirow{2}{*}{$\begin{array}{c}2.1 \\
\text { Regulamentação } \\
\text { das comissões } \\
\text { de farmácia e } \\
\text { terapêutica na } \\
\text { instituição }\end{array}$} & $\begin{array}{l}\text { Divulgação da resolução com } \\
\text { enfoque nos fluxos e rotinas da CFT }\end{array}$ & Coord. AF & $\begin{array}{l}\text { Assessoria de } \\
\text { Comunicação }\end{array}$ & 7 dias & \multirow{5}{*}{$\begin{array}{l}\text { Formação das } \\
\text { CFT, treinamento } \\
\text { e posse dos } \\
\text { membros } \\
\text { [2 meses] }\end{array}$} \\
\hline & & $\begin{array}{l}\text { Indicação de membros para } \\
\text { compor as CFT }\end{array}$ & Coord. AF & $\begin{array}{l}\text { Gestores das } \\
\text { unidades }\end{array}$ & 15 dias & \\
\hline & \multirow{3}{*}{$\begin{array}{l}2.2 \text { Formação } \\
\text { das comissões, } \\
\text { nomeação de } \\
\text { membros e início } \\
\text { dos trabalhos }\end{array}$} & $\begin{array}{l}\text { Posse dos membros e assinatura de } \\
\text { declaração de conflito de interesses }\end{array}$ & Coord. AF & - & 7 dias & \\
\hline & & $\begin{array}{l}\text { Definição de atribuiç̃oes entre } \\
\text { os membros e cronograma de } \\
\text { reuniões }\end{array}$ & $\begin{array}{l}\text { Presidentes } \\
\text { das CFT }\end{array}$ & $\begin{array}{l}\text { Membros das } \\
\text { CFT }\end{array}$ & 7 dias & \\
\hline & & $\begin{array}{l}\text { Treinamento dos membros sobre } \\
\text { avaliação de tecnologias em saúde }\end{array}$ & $\begin{array}{l}\text { Presidentes } \\
\text { das CFT }\end{array}$ & $\begin{array}{l}\text { Assessoria de } \\
\text { Comunicação }\end{array}$ & 21 dias & \\
\hline
\end{tabular}




\begin{tabular}{|c|c|c|c|c|c|c|}
\hline \multirow{2}{*}{ OBJETIVOS } & \multirow{2}{*}{ OPERAÇÕES } & \multirow{2}{*}{ AÇÕES ESPECÍFICAS } & \multicolumn{2}{|c|}{$\begin{array}{l}\text { RESPONSABILIDADE E } \\
\text { CENTRALIDADE }\end{array}$} & \multirow{2}{*}{ PRAZO } & \multirow{2}{*}{$\begin{array}{c}\text { INDICADOR } \\
\text { DE AVALIAÇÃO } \\
\text { [tempo } \\
\text { estimado] }\end{array}$} \\
\hline & & & $\begin{array}{c}\text { ATOR } \\
\text { PRINCIPAL }\end{array}$ & PARCEIROS & & \\
\hline \multirow{6}{*}{$\begin{array}{l}\text { 3. Elaborar } \\
\text { relações de } \\
\text { medicamentos } \\
\text { essenciais de } \\
\text { acordo com nível } \\
\text { de complexidade } \\
\text { do serviço }\end{array}$} & \multirow{3}{*}{$\begin{array}{c}\text { 3.1 Seleção dos } \\
\text { medicamentos } \\
\text { essenciais pelas } \\
\text { CFT }\end{array}$} & $\begin{array}{l}\text { Elaboração de lista inicial baseada } \\
\text { no histórico de utilização das } \\
\text { unidades }\end{array}$ & Coord. AF & - & 15 dias & \multirow{6}{*}{$\begin{array}{l}\text { Publicação das } \\
\text { relações de } \\
\text { medicamentos } \\
\text { por nível de } \\
\text { complexidade } \\
\text { [8 meses] }\end{array}$} \\
\hline & & $\begin{array}{l}\text { Revisão dos medicamentos de } \\
\text { acordo com grupo terapêutico }\end{array}$ & $\begin{array}{l}\text { Presidentes } \\
\text { das CFT }\end{array}$ & $\begin{array}{l}\text { Membros das } \\
\text { CFT }\end{array}$ & 120 dias & \\
\hline & & $\begin{array}{l}\text { Elaboração das listas com itens } \\
\text { selecionados pelas CFT por serviço }\end{array}$ & $\begin{array}{l}\text { Presidentes } \\
\text { das CFT }\end{array}$ & $\begin{array}{l}\text { Membros das } \\
\text { CFT }\end{array}$ & 15 dias & \\
\hline & \multirow{3}{*}{$\begin{array}{l}3.2 \text { Validação } \\
\text { das listas de } \\
\text { medicamentos } \\
\text { selecionados }\end{array}$} & $\begin{array}{l}\text { Consulta pública da lista } \\
\text { selecionada }\end{array}$ & Coord. AF & $\begin{array}{l}\text { Assessoria de } \\
\text { Comunicação }\end{array}$ & 30 dias & \\
\hline & & $\begin{array}{l}\text { Apreciação das contribuiç̃ões e } \\
\text { julgamentos finais das CFT }\end{array}$ & $\begin{array}{l}\text { Presidentes } \\
\text { das CFT }\end{array}$ & $\begin{array}{l}\text { Membros das } \\
\text { CFT }\end{array}$ & 15 dias & \\
\hline & & $\begin{array}{l}\text { Institucionalização efetiva das } \\
\text { listas de medicamentos (compras, } \\
\text { serviço, outros) }\end{array}$ & $\begin{array}{l}\text { Gestão dos } \\
\text { Serviços }\end{array}$ & $\begin{array}{l}\text { Membros das } \\
\text { CFT }\end{array}$ & 45 dias & \\
\hline
\end{tabular}

Fonte: Resultados da pesquisa.

Como pode ser visualizado nas matrizes elaboradas, o processo de implantação do PES girou em torno de três objetivos principais: 1) a regulamentação de fluxos e procedimentos para a seleção de medicamentos; 2) a organização das CFT; 3) a elaboração da lista de medicamentos essenciais. No quadro 5 estão descritos os principais resultados obtidos de acordo com cada objetivo proposto.

Quadro 5

Painel de resultados obtidos mediante o PES

\begin{tabular}{|c|c|}
\hline RESULTADOS GERAIS & RESULTADOS ESPECÍFICOS \\
\hline \multirow{6}{*}{$\begin{array}{l}\text { 1. Elaboração e publicação } \\
\text { de resolução que } \\
\text { regulamenta o } \\
\text { processo de seleção de } \\
\text { medicamentos }\end{array}$} & $\begin{array}{l}\text { 1.1 Formação de Grupo de Trabalho para avaliação da assistência farmacêutica e elaboração da proposta de } \\
\text { regulamento para seleção de medicamentos }\end{array}$ \\
\hline & 1.2 Elaboração de normas para criação e funcionamento das Comissões de Farmácia e Terapêutica (CFT) \\
\hline & $\begin{array}{l}\text { 1.3 Definição de indicadores para avaliação de medicamentos com aspectos de saúde baseada em evidências } \\
\text { e farmacoeconomia }\end{array}$ \\
\hline & $\begin{array}{l}\text { 1.4 Definição de critérios para avaliação de aspectos éticos dos membros da CFT e da relação destes e dos } \\
\text { profissionais de saúde do serviço com o mercado farmacêutico }\end{array}$ \\
\hline & 1.5 Avaliação de aspectos jurídicos relacionados à incorporação de medicamentos \\
\hline & 1.6 Publicação da resolução que regulamenta o processo de seleção de medicamentos \\
\hline \multirow{5}{*}{$\begin{array}{l}\text { 2. Organização das } \\
\text { comissões de farmácia e } \\
\text { terapêutica por nível de } \\
\text { complexidade dos serviços }\end{array}$} & 2.1 Divulgação do processo de seleção em implantação e indicação de membros estratégicos da criação das CFT \\
\hline & $\begin{array}{l}\text { 2.2 Criação de cinco CFT de acordo com nível de complexidade dos serviços: CFT1 (hospitais locais); CFT2 } \\
\text { (hospitais regionais); CFT3 (unidades de saúde materno-neonatal); CFT4 (hospital geral de grande porte); CF5 } \\
\text { (serviço de atendimento móvel pré-hospitalar) }\end{array}$ \\
\hline & $\begin{array}{l}2.3 \text { Assinatura de termos de declaração de interesses pelos membros, definição de atribuições e cronograma } \\
\text { de reuniões das CFT }\end{array}$ \\
\hline & $\begin{array}{l}\text { 2.4 Realização de treinamento dos membros da CFT: Seminário sobre Avaliação de Tecnologias em Saúde } \\
\text { (ATS) }\end{array}$ \\
\hline & $\begin{array}{l}2.5 \text { Realização de } 44 \text { reuniões de CFT com periodicidade variando de uma vez por semana a uma vez por } \\
\text { mês }\end{array}$ \\
\hline
\end{tabular}




\begin{tabular}{|c|c|}
\hline RESULTADOS GERAIS & RESULTADOS ESPECÍFICOS \\
\hline \multirow{7}{*}{$\begin{array}{l}\text { 3. Elaboração e implantação } \\
\text { de listas de medicamentos } \\
\text { essenciais }\end{array}$} & $\begin{array}{l}\text { 3.1 Revisão dos itens do histórico pelos membros da CFT baseada na experiência clínica dos membros, em } \\
\text { aspectos de saúde baseada em evidências e farmacoeconomia }\end{array}$ \\
\hline & $\begin{array}{l}\text { 3.2 Implantação de formulários e critérios para solicitação de inclusão, exclusão e substituiçã̃o de medicamentos } \\
\text { das listas-padrão }\end{array}$ \\
\hline & 3.3 Realização de consulta pública sobre medicamentos selecionados pela CFT \\
\hline & $\begin{array}{l}\text { 3.4 Participação de } 54 \text { especialistas durante todo o processo com uma média de participação de } 10 \text { membros } \\
\text { por reunião }\end{array}$ \\
\hline & 3.5 Elaboração de 15 pareceres técnico-científicos sobre medicamentos da lista (demanda interna ou externa) \\
\hline & $\begin{array}{l}\text { 3.6 Elaboração da primeira lista de medicamentos selecionados composta da junção de } 5 \text { listas menores } \\
\text { elaboradas de acordo com cada CFT implantada (ver item 2.3) }\end{array}$ \\
\hline & $\begin{array}{l}\text { 3.7 Redução de } 27,8 \% \text { dos medicamentos, passando de } 716 \text { itens do histórico inicial levantado para } 516 \text { itens } \\
\text { na lista oficial validada pela gestão da instituição }\end{array}$ \\
\hline
\end{tabular}

Fonte: Resultados da pesquisa.

\subsection{Regulamentação de fluxos e procedimentos para a seleção de medicamentos}

Na execução do primeiro objetivo, por meio da elaboração de uma resolução específica, visouse garantir duas questões fundamentais: a padronização de critérios de seleção baseada em evidências científicas e o combate às interferências mercadológicas.

Para tornar o processo de seleção de medicamentos mais efetivo, nos últimos anos, os critérios utilizados deixaram de se basear apenas na experiência clínica dos membros e passaram a ser pautados fortemente em evidências científicas. Revisões da literatura já demonstram que a maioria dos trabalhos $(62,5 \%)$ relacionados à seleção de medicamentos coloca como prerrequisitos essenciais para esta atividade o embasamento em métodos de saúde baseada em evidências e farmacoeconomia (Wannmacher, 2006; Santana et al., 2014).

Crescente também é a preocupação com a transparência do processo e a isenção dos membros dos comitês que confeccionam as listas, os quais devem aliar competência técnica a total ausência de conflito de interesses e profundo senso ético (Wannmacher, 2006). Os membros das CFT das unidades analisadas foram orientados sobre os aspectos éticos relacionados à participação desse comitê e assinaram termos onde declaram isenção de conflito de interesses antes da posse dos seus cargos. A OMS preconiza que se deve exigir de todos os membros do comitê a assinatura desse tipo de declaração, a fim de comprometê-los com os princípios de trabalho e ética da CFT (WHO, 2003).

\subsection{Organização das CFT}

Após definição dos critérios técnicos e éticos para a seleção de medicamentos, as ações do PES ficaram concentradas na organização efetiva das CFT. As 13 unidades-alvo do estudo foram agrupadas de acordo com suas características de porte e serviços ofertados em cinco CFT com membros representativos dos serviços de farmácia e enfermagem, da gestão e das diversas 
especialidades médicas presentes nos hospitais. Estudos internacionais relatam o incentivo ou predomínio de CFT por unidade hospitalar, talvez no sentido de preservar as características e necessidades individuais (Mittmann e Knowles, 2009; Puigventós et al., 2010). Estudo semelhante nacional optou pela fusão de comitês para organização de CFT única em nível estadual, por entender que esse modelo favorece as análises de comparação de itens e pode garantir a discussão de tratamentos não ofertados pelos comitês individuais (Magarinos-Torres et al., 2013). A organização num formato intermediário a esses modelos visou contemplar necessidades locais, agrupando unidades com perfis semelhantes, porém garantindo resultados comparados e consensuais entre os comitês por meio de um comando único do processo e intensa troca de informações.

A OMS estabelece que um dos resultados esperados para promoção do uso racional de medicamentos pelos profissionais de saúde e consumidores dos países em desenvolvimento é a promoção das CFT em nível institucional, municipal, estadual e nacional (WHO, 2004).

Foram realizadas 44 reuniões das CFT com periodicidade variando de uma vez por semana a uma vez por mês, com a média de 10,6 $\pm 2,7$ participantes por reunião. Apesar de não haver uma estatística nacional de base governamental, um estudo realizado com 250 hospitais públicos e privados de diversas regiões brasileiras mostrou que apenas 29 hospitais haviam implantado uma CFT. Ainda assim, em apenas nove desses hospitais a CFT funcionava regularmente, ou seja, com no mínimo uma reunião a cada dois meses (Osório e Castilho, 2004). Outros estudos demonstram a media de 5,1 reuniões nos hospitais espanhóis (DuránGarcía et al., 2011) e 9,7 reuniões em hospitais americanos (Mannebach et al., 1999).

\subsection{Implantação da lista de medicamentos}

Após a organização efetiva das CFT, as atividades do plano seguiram para a construção efetiva da primeira relação de medicamentos essenciais dos hospitais públicos sergipanos.

Por entender que a representação das diferentes categorias é essencial para a qualidade do processo, na elaboração da lista participaram do processo de avaliação 54 colaboradores, sendo, destes, 20 profissionais médicos de diversas especialidades, 15 farmacêuticos e 14 enfermeiros. A participação e o envolvimento dos profissionais da equipe de saúde diretamente no processo de seleção contribuem para que estes se sintam corresponsáveis com a relação de medicamentos selecionados, além de acrescentar valor técnico ao trabalho. Essa participação pode contribuir com a aceitação da lista e com a prescrição dos medicamentos contemplados (Oliveira, Grochocki e Pinheiro, 2011).

Além da presença de representantes das diversas categorias, foi garantida a participação ampla da comunidade hospitalar por meio de fluxos padronizados de inclusão e exclusão de itens da lista. As propostas elaboradas pelas CFT também passaram por processo de consulta pública, por meio do site da instituição, onde todos os profissionais poderiam opinar sobre os itens pré-selecionados. Estudos nacionais (Magarinos-Torres et al., 2011) e internacionais (Wettermark et al., 2005) relatam a pouca participação de prescritores e demais profissionais 
de saúde durante esse processo, no entanto, neste estudo os atores consideraram que essa etapa do processo está relacionada com a legitimação da atividade de seleção de medicamentos.

Outro indicador de qualidade alcançado é a fundamentação das decisões baseadas na evidência científica e não apenas na experiência clínica dos profissionais envolvidos no cuidado. Durante a construção da relação de medicamentos selecionados foram elaborados 15 pareceres técnico-científicos para documentar e fundamentar as alterações que mais possuíam impacto econômico e/ou assistencial. Esses pareceres seguiam sempre a lógica de elaboração e revisão por dois membros da CFT, apreciação e homologação em reunião ordinária do comitê. Os pareceres técnico-científicos (PTC) são uma ferramenta de suporte à gestão e à decisão, baseada na mesma racionalidade que envolve uma ATS, contudo com execução e conteúdo mais simplificados. Embora envolvam, via de regra, uma revisão da literatura menos extensa e abrangente quando comparados com uma revisão sistemática, por serem de execução e elaboração mais rápidas, os PTC devem representar um relato sistematizado e abrangente do conhecimento possível de ser fornecido neste contexto, contribuindo para qualificar as decisões a serem tomadas (Brasil, 2009a).

Ao final do PES foi concluída e homologada a primeira de lista de medicamentos essenciais, que estava subdivida em cinco outras listas estruturadas a partir do trabalho das cinco CFT organizadas e que representavam as necessidades das unidades de acordo com seu grau de serviços ofertados. Ao se comparar com a relação de medicamentos adquiridos antes do início dos trabalhos da CFT, baseada no histórico de consumo e pedidos de compra, observouse uma redução de $27,8 \%$ no número total de medicamentos utilizados: de 716 itens na relação inicial para 516 itens, o que representou uma redução de aproximadamente 12\% da demanda de recursos dos serviços avaliados.

A exclusão de itens não essenciais ou de efetividade e segurança questionável representa grande contribuição aos cuidados em saúde. A diminuição no número de itens é um ganho para a assistência farmacêutica hospitalar, pois contribui para a melhora da logística, do fornecimento de informação e do acompanhamento do uso pela farmácia hospitalar. Além disso, os gastos totais tendem a diminuir com o aumento do volume de compra de um mesmo item, por economia de escala (Oliveira et al., 2007; Reis e Perini, 2008; Magarinos-Torres et al., 2011).

Além da redução do número global de itens, foram também incluídos 25 medicamentos para tratamento de determinadas enfermidades que não estavam contempladas ou possuíam alternativas de menor efetividade e custo-benefício, como medicamentos para a hipertermia maligna, trombolíticos utilizados no infarto agudo do miocárdio, anestésicos gerais e locais, medicamentos para tratamento da dor, anticorpos monoclonais, determinados antineoplásicos, dentre outros. Essas inclusões, fundamentadas pela ótica científica, beneficiam os usuários do serviço que podem contar com ferramentas mais efetivas.

As intervenções realizadas por meio do PES foram decisivas para a institucionalização de um processo estruturado de incorporação de medicamentos — um dos mais visíveis desafios globais na atenção à saúde (Wannmacher, 2010; Greene, 2008) —, que permaneceu vigente nos hospitais mesmo após a finalização do estudo. 
Além de constantemente atualizados e reposicionados, os resultados precisam ser mantidos. A sustentabilidade refere-se à probabilidade de um programa ser capaz de continuar suas atividades operacionais e manter seus resultados ao longo do tempo. Para tanto, questões como estabilidade financeira e a permanência de direção são importantes, mas com certeza a legitimidade das ações e o estabelecimento dos vínculos de apoio com os diversos atores envolvidos são fundamentais (Ocde, 2002; Brasil, 2009b).

A garantia da sustentabilidade de programas ou ações pode ser definida por rotinas organizacionais ou obedecer a padrões institucionais oficiais, que dão origem a rotinas padronizadas mais duráveis. Rotinas padronizadas e institucionalizadas, planejadas e executadas de forma participativa e com análise estratégica e situacional, como as desenvolvidas neste trabalho, são mais duráveis, resistem melhor a mudanças cotidianas, mas precisam ser renovadas de tempos em tempos para que os programas sobrevivam (Pluye, Potvin e Denis, 2004).

\section{Considerações finais}

Diante dos dados relatados é possível perceber a complexidade organizacional envolvida na implantação de atividades de seleção de medicamentos e os desafios que devem ser enfrentados para que esse processo seja exitoso. Pela proposta deste estudo, o PES demonstra ser uma ferramenta aplicável à correta identificação dos problemas locais, para o planejamento adequado das etapas de seleção, para a previsão das dificuldades de implantação e para o acompanhamento e avaliação da política de medicamentos essenciais.

O sucesso obtido no estabelecimento e funcionamento das CFT no estado do Sergipe é um exemplo importante para o SUS, que denota sua capacidade de organização e de trabalho com racionalidade e metodologia técnica e cientificamente embasada.

A experiência aqui descrita demonstra que, além da vontade política, os preceitos de governo de Carlos Matus de participação, transparência, negociação entre diferentes setores e interesses, aplicados no PES, colaboram para a superação das barreiras operacionais comumente imputadas à seleção de medicamentos em serviços públicos e, especialmente, à aceitação e incorporação das listas padronizadas de medicamentos. De tal forma, tratou-se a seleção de medicamentos não como apenas um serviço técnico-operacional de assistência farmacêutica, mas como um processo que precisa ser conduzido, aplicando o conceito de gestão como processo técnico, político e social.

\section{Referências}

ALMEIDA, Eurivaldo S. et al. Planejamento e programação em saúde. In: WESTPHAL, Marcia F.; ALMEIDA, Eurivaldo S. (Org.). Gestão de serviços de saúde. São Paulo: Edusp, 2001. p. 255-272. 
ARTMANN, Elizabeth; AZEVEDO, Creusa S.; SÁ, Marilene C. Possibilidades de aplicação do enfoque estratégico de planejamento no nível local de saúde: análise comparada de duas experiências. Cadernos de Saúde Pública, v. 13, n. 4, p. 723-740, 1997.

BERNARDINO, Elizabeth; OLIVEIRA, Elda; CIAMPONE, Maria H. T. Preparando enfermeiros para o SUS: o desafio das escolas formadoras. Revista Brasileira de Enfermagem, v. 59, n. 1, p. 36-40, 2006.

BRASIL. Ministério da Saúde. Diretrizes metodológicas: elaboração de pareceres técnico-científicos. 2. ed. Brasília, 2009a.

BRASIL. Ministério da Saúde. Secretaria-Executiva. Subsecretaria de Planejamento e Orçamento. Sistema de planejamento do SUS: uma construção coletiva: instrumentos básicos. Brasília, 2009b.

CEBRIM. Centro Brasileiro de Informação sobre Medicamentos. Boletim Farmacoterapêutica, v. 8, n. 2, p. 1-4, 2003.

CONTRADIOPOULOS, André P. A avaliação na área da saúde: conceitos e métodos. In: HARTZ, Zulmira M. A. (Org.). Avaliação em saúde: dos modelos conceituais à prática na análise da implantação de programas. Rio de Janeiro: Editora Fiocruz, 1997. p. 29-47.

DURÁN-GARCÍA, Esther et al. Literature review on the structure and operation of pharmacy. International Journal of Clinical Pharmacy, v. 33, n. 3, p. 475-483, 2011.

GIOVANELLA, Ligia. As origens e as correntes atuais do enfoque estratégico em planejamento de saúde na América Latina. Cadernos de Saúde Pública, v. 7, n. 1, p. 26-44, 1991.

GREENE, Jeremy A. When did medicines become essential. Bulletin of the World Health Organization, v. 88, n. 7, p. 483, 2008.

ISHIKAWA, Kaoru. Controle de qualidade total: à maneira japonesa. Rio de Janeiro: Campos, 1993.

KRAUSS-SILVA, Letícia. Avaliação tecnológica e análise custo-efetividade em saúde: a incorporação de tecnologias e a produção de diretrizes clínicas para o SUS. Ciência \& Saúde Coletiva, v. 8, n. 2, p. 501-520, 2003.

KRAUSS-SILVA, Letícia. Avaliação tecnológica em saúde: questões metodológicas e operacionais. Cadernos de Saúde Pública, v. 20, n. 2, p. 199-207, 2004.

LEITE, Silvana N.; GUIMARÃES, Maria C. L. Gestão da assistência farmacêutica. In: BRASIL. Gestão da assistência farmacêutica [Recurso eletrônico] / Universidade Federal de Santa Catarina, Universidade Aberta do SUS. Florianópolis: UFSC, 2011.

MAGARINOS-TORRES, Raquel et al. Estruturação da assistência farmacêutica: plano de ação para a seleção de medicamentos essenciais. Cadernos de Saúde Coletiva, v. 21, n. 2, p. 188-196, 2013.

MAGARINOS-TORRES, Raquel et al. Vivenciando a seleção de medicamentos em hospital de ensino. Revista Brasileira de Educação Médica, v. 35, n. 1, p. 77-85, 2011.

MANNEBACH, Mark A. et al. Activities, functions, and structure of pharmacy and therapeutics 
committees in large teaching hospitals. American Journal of Health-System Pharmacy, v. 56, n. 7, p. 622-628, 1999.

MARQUES, Dirce C.; ZUCCHI, Paola. Comissões farmacoterapêuticas no Brasil: aquém das diretrizes internacionais. Revista Panamericana de Salud Pública, v. 19, n. 1, p. 58-68, 2006.

MATUS, Carlos. Política, planejamento \& governo. Brasília: Ipea, 1996.

MELLEIRO, Marta M.; TRONCHIN, Daisy M. R.; CIAMPONE, Maria H. T. O planejamento estratégico situacional no ensino do gerenciamento em enfermagem. Acta Paulista de Enfermagem, v. 18, n. 2, p. 165-171, 2005.

MINAYO, Maria C. S.; SANCHES, Odécio. Qualitativo-quantitativo: oposição ou complementaridade? Cadernos de Saúde Pública, v. 9, n. 3, p. 239-248, 1993.

MITTMANN, Nicole; KNOWLES, Sandra. A survey of pharmacy and therapeutic committees across of Canada: scope and responsibilities. Canadian Journal of Clinical Pharmacology, v. 16, n. 1, p. 171-177, 2009.

OCDE. Organization for Economic Co-operation and Development. Working party on aid evaluation. Glossary of key terms in evaluation and results based management. Paris: Development Co-Operation Directorate; Ocde, 2002. Disponível em: <www.oecd.org/dac/evaluation>. Acesso em: 17 abr. 2013.

OLIVEIRA, Jardel C.; GROCHOCKI, Mônica H. C. G.; PINHEIRO, Rafael M. Seleção de medicamentos. In: BRASIL. Gestão da assistência farmacêutica [Recurso eletrônico]/Universidade Federal de Santa Catarina, Universidade Aberta do SUS. Florianópolis: UFSC, 2011.

OLIVEIRA, Maria A.; BERMUDEZ, Jorge A. Z.; OSORIO-DE-CASTRO, Claudia G. S. Assistência farmacêutica e acesso a medicamentos. Rio de Janeiro: Editora Fiocruz, 2007. (Temas em Saúde).

OSÓRIO, Claudia G. S.; CASTILHO, Selma R. Diagnóstico da farmácia hospitalar no Brasil. Rio de Janeiro: ENSP/Fiocruz, 2004.

PLUYE, Pierre; POTVIN, Louise; DENIS, Jean-Louis. Making public health programs last: conceptualizing sustainability. Evaluation and Program Planning, v. 27, n. 2, p. 121-133, 2004.

PUIGVENTÓS, Francesc et al. Structure and procedures of the pharmacy and therapeutic committees in Spanish hospitals. Pharmacy World and Science, v. 32, n. 6, p. 767-775, 2010.

REIS, Adriano M. M.; PERINI, Edson. Desabastecimento de medicamentos: determinantes, consequências e gerenciamento. Ciência e Saúde Coletiva, v. 13, n. sup. 0, p. 603-610, 2008.

SANTANA, Rafael S. et al. Indicadores da seleção de medicamentos em sistemas de saúde: uma revisão integrativa. Revista Panamericana de Salud Publica, v. 35, n. 3, p. 228-234, 2014.

SANTOS, Vânia C. C. As análises econômicas na incorporação de tecnologias em saúde: reflexões sobre a experiência brasileira. Dissertação (mestrado em saúde pública) — Fundação Oswaldo Cruz, Rio de Janeiro, 2010.

SILVA, Hudson P.; PETRAMALE, Clarisse A.; ELIAS, Flavia T. S. Avanços e desafios da política nacional de gestão de tecnologias em saúde. Revista de Saúde Pública, v. 46, n. supl., p. 83-90, 2012. 
TONI, Jackson. O que é o planejamento estratégico situacional? Revista Espaço Acadêmico, n. 32, jan. 2004. Disponível em: <www.espacoacademico.com.br/032/32ctoni.htm>. Acesso em: 12 nov. 2012.

VELÁSQUEZ, German. Impacto económico del uso racional de medicamentos. In: BERMÚDEZ, Jorge A. Z.; BOMFIM, José R. A. (Org.). Medicamentos e a reforma do setor saúde. São Paulo: Hucitec; Sobravime, 1999. p. 29-34.

WANNMACHER, Lenita. Medicamentos essenciais: vantagens de trabalhar com este contexto. Boletim OPAS: Uso Racional de Medicamentos - Temas Selecionados, v. 3, n. 2, p. 1-6, 2006.

WANNMACHER, Lenita. Seleção de medicamentos essenciais: propósitos e consequências. Revista Tempus Actas Saúde Coletiva, v. 4, n. 3, p. 23-29, 2010.

WEEKES, Lynn M.; BROOKS, Colleen. Drug and Therapeutics Committees in Australia: expected and actual performance. British Journal of Clinical Pharmacology, v. 42, n. 5, p. 551-557, 1996.

WETTERMARK, Björn et al. A study of adherence to drug recommendations by providing feedback of outpatient prescribing patterns to hospital specialists. Pharmacoepidemiology Drug and Safety, v. 14, n. 8, p. 579-588, 2005.

WHO. World Health Organization. $18^{\text {th }}$ Model list of essential medicines, 2013. Disponível em: <www. who.int/medicines/publications/essentialmedicines/ 18th_EML_Final_web_8Jul13.pdf $>$. Acesso em: 10 maio 2014.

WHO. World Health Organization. Drug and therapeutics committees: a practical guide. Genebra: Department of Essential Drugs and Medicines Policy, 2003.

WHO. World Health Organization. Medicines strategy: countries at the core. Genebra, 2004.

WHO. World Health Organization. Report on the $12^{\text {th }}$ Expert Committee on the Selection and Use of Essential Medicines. Technical Report Series, n. 914. Genebra: 2002.

Rafael Santos Santana é doutorando em ciências farmacêuticas pela Universidade de Brasília (UnB). Consultor técnico do Ministério da Saúde. E-mail: rafaels.santana1@gmail.com.

Iza Maria Fraga Lobo é doutora em medicina e saúde pela Universidade Federal da Bahia (Ufba). Gerente de Risco do Hospital Universitário da Universidade Federal de Sergipe (UFS). E-mail: izalobo@ globo.com.

Thais Rodrigues Penaforte é doutora em medicamentos e cosméticos pela Universidade de São Paulo (USP). Professora da Universidade Federal da Bahia (Ufba). E-mail: thaisrpenaforte@gmail.com.

Silvana Nair Leite é doutora em saúde pública pela Universidade de São Paulo (USP). Professora da Universidade Federal de Santa Catarina (UFSC). E-mail: silvana.nair@hotmail.com.

Wellington Barros Silva é doutor em educação científica e tecnológica pela Universidade Federal de Santa Catarina (UFSC). Professor da Universidade Federal de Sergipe (UFS). E-mail: wbarrosdasilva@ gmail.com. 